\title{
Sub-GHz LPWAN network coexistence, management and virtualization: an overview and open research challenges
}

\author{
Eli De Poorter', Jeroen Hoebeke', Matthias Strobbe', Ingrid Moerman', Steven \\ Latré ${ }^{2}$ Maarten Weyn ${ }^{2}$, Bart Lannoo ${ }^{2}$ and Jeroen Famaey \\ 'Ghent University - imec, IDLab, Belgium \\ \{eli.depoorter, jeroen.hoebeke, matthias strobbe \\ ingrid.moerman\} eugent.be \\ ${ }^{2}$ University of Antwerp - imec, IDLab, Belgium \\ \{steven.latre, maarten.weyn, bart.lannoo, \\ jeroen.famaey\} @uantwerpen.be
}

\begin{abstract}
The IoT domain is characterized by many applications that require lowbandwidth communications over a long range, at a low cost and at low power. LPWANs (Low Power Wide Area Networks) fulfill these requirements by using sub-GHz radio frequencies (typically 433 or $868 \mathrm{MHz}$ ) with typical transmission ranges in the order of 1 up to 50 kilometers. As a result, a single base station can cover large areas and can support high numbers of connected devices ( $>1000$ per base station). Notorious initiatives in this domain are LoRa, Sigfox and the upcoming IEEE 802.11ah (or "HaLow") standard. Although these new technologies have the potential to significantly impact many IoT deployments, the current market is very fragmented and many challenges exists related to deployment, scalability, management and coexistence aspects, making adoption of these technologies difficult for many companies. To remedy this, this paper proposes a conceptual framework to improve the performance of LPWAN networks through in-network optimization, cross-technology coexistence and cooperation and virtualization of management functions. In addition, the paper gives an overview of state of the art solutions and identifies open challenges for each of these aspects.
\end{abstract}

Keywords: sub-GHz networks, LPWAN, LoRa, SigFox, IEEE 802.11ah, DASH7, coexistence, network management, virtualization, scalability, QoS, energy efficiency.

\section{$1 \quad$ Introduction}

The IoT domain is characterized by many applications that require low-bandwidth communications over a long range, at a low cost and at low power. Recent technology developments have given rise to novel 'SIM-less' radio technologies with much larger coverage ranges. These cheap, low-power wide area IoT networks, often referred to as Low Power Wide Area Networks or LPWANs, try to fill in an existing market 
gap allowing devices to be connected without requiring significant infrastructure investments. LPWAN technologies have several defining characteristics. (i) They have very low power consumption, resulting in a battery life of up to 15 years for some applications. (ii) They support only short information exchanges, often 100 bytes or less. (iii) They have very low device unit costs; the connectivity module will eventually cost less than a few dollar. And (iv) they are designed to have good coverage indoors and outdoors. Due to the use of sub-GHz radio frequencies (typically 433 or $868 \mathrm{MHz}$ in Europe), a single LPWAN base station has a large coverage area, with a range from 1 up to $50 \mathrm{~km}$. This way, a single base station can support high numbers of connected devices (> 1000 per base station). Although the size of and frequency of information exchanges is limited, this disadvantage is off-set by the fact that a single sub- $\mathrm{GHz}$ base station can cover an area of multiple square kilometers, allowing cost-effective installations of large IoT deployments.

Currently, several sub-GHz technologies are being promoted simultaneously, all of which use the same (limited) wireless spectrum. Notorious initiatives in this domain are LoRa, SigFox, IEEE802.15.4g and the upcoming IEEE 802.11ah (or "HaloW") standard. However, as several LPWAN technologies are entering the market simultaneously, several global trends start to emerge.

1. The LPWAN landscape is currently very fragmented with many different network operators. As a result, it is often not clear which technology (if any current technology) is the most appropriate one for a certain application domain (e.g. for critical applications with stringent QoS or reliability constraints).

2. In addition, many of these technologies are still in their infancy, and optimizations regarding a.o. quality of service, roaming, and service management are still lacking.

3. Finally, since the amount of available spectrum is much smaller and the propagation ranges much larger, these technologies will cause interference at much larger scale, leading to severe inter-technology and inter-operator interference. If left unchecked the unlicensed sub-1GHz bands will soon be congested and unreliable.

As such, the presence of these different LPWAN solutions creates coexistence problems, threatening the creation of a sustainable market consisting of reliable, interoperable LPWAN technologies. This paper outlines several mitigating steps that can overcome these risk. To this end, the paper proposes an advanced coordination framework to uniformly manage and optimize an ecosystem of coexisting wireless sub-GHz LPWANs and identifies open research challenges that need to be solved to realize this overall vision.

The remainder of this paper is structured as follows. Section 2 gives an overview of several recent LPWAN technologies. Section 3 proposes an architecture to manage and optimize heterogeneous LPWAN networks and discusses future challenges at different levels. The current state of the art and future research directions related to optimizing 
LPWAN networks within a single technology (.e.g scalability, deterministic networks and energy efficiency) are discussed in Section 4. Afterwards, Section 5 discusses current approaches for LPWAN cross-technology coexistence. Section 6 gives an overview of current approaches and limitations for efficient management of LPWAN networks, including virtualization, self-optimization and remote reconfiguration and over-the-air update solutions. Finally, Section 7 concludes the paper.

\section{LPWAN technologies}

The sections below discuss the current state-of-the-art approaches for LPWAN technologies. Many civic, industrial and other IoT applications need to operate over a greater territory than existing technologies that operate in the $2.4 \mathrm{GHz}$ or $5 \mathrm{GHz}$ ISM band can handle. To fill in this gap, a number of low-power wide-area networking alternatives have arisen that offer an extended range at low energy consumption by operating in unlicensed sub-GHz frequency bands (the $915 \mathrm{MHz}$ ISM band in the US or the $868 \mathrm{MHz}$ band for Short Range Devices in the EU). Table 1gives a summary of several noteworthy \& representative technologies together with several key characteristics.

Table 1. Overview of notew orthy LPWAN technologies in the sub-GHz bands

\begin{tabular}{|l|c|c|c|c|c|}
\hline Technology & Bandwidth & Data rate & Range & Multihop & Remarks \\
\hline LoRaWAN & $125 \mathrm{kHz} / 250 \mathrm{kHz}$ & $\begin{array}{c}250 \mathrm{bps}- \\
5.5 \mathrm{kbps} / \\
11 \mathrm{kbps} / \\
50 \mathrm{kbps}\end{array}$ & $2-15 \mathrm{~km}$ & No & $\begin{array}{c}\text { Open system } \\
\text { specification }\end{array}$ \\
\hline IEEE \\
$\mathbf{8 0 2 . 1 1 a h}$
\end{tabular}

In the following subsections, we shortly introduce the selected LPWAN technologies.

\subsection{LoRaWAN}

LoRa is a physical layer technology for creating long range communication links. It makes use of a novel radio modulation technique, Chirp Spread Spectrum (CSS) that 
has a very high sensitivity and thus increased communication range. LoRaWAN defines the communication protocol and system architecture for the network, on top of the LoRa physical layer. In Europe, LoRaWAN defines ten channels. Eight of these are $125 \mathrm{kHz}$ LoRa channels which, depending on the used spreading factor, offer data rates from $250 \mathrm{bps}$ to $5.5 \mathrm{kbps}$. By dynamically adapting the spreading factor a trade-off between data rate and range can be achieved (ADR or Adaptive Data Rate). In addition, there is also a single high data rate $250 \mathrm{kHz}$ LoRa channel at $11 \mathrm{kbps}$ and a single FSK channel at $50 \mathrm{kbps}[1]$.

The network architecture defined by LoRaWAN is a star on star architecture. Uplink data originating from end devices is received by one or multiple gateways. These gateways act as transparent (non-intelligent) bridges, relaying the data to a cloud-based network server. In case downlink traffic is available, the cloud server decides which gateway has the best connectivity with the end device, and the downlink traffic is transmitted from this gateway. As such, all intelligence resides in this network server, which manages the network, filters redundant received packets, performs security checks, schedules acknowledgments through the optimal gateway, performs adaptive data rate, etc. Currently, 3 devices classes (A, B and C) have been defined (Figure 1): class A allows download traffic right after an upload slot, class B schedules separate upload slots and class $\mathrm{C}$ continuously allows download traffic, thereby trading in battery lifetime for lower downlink communication latency.

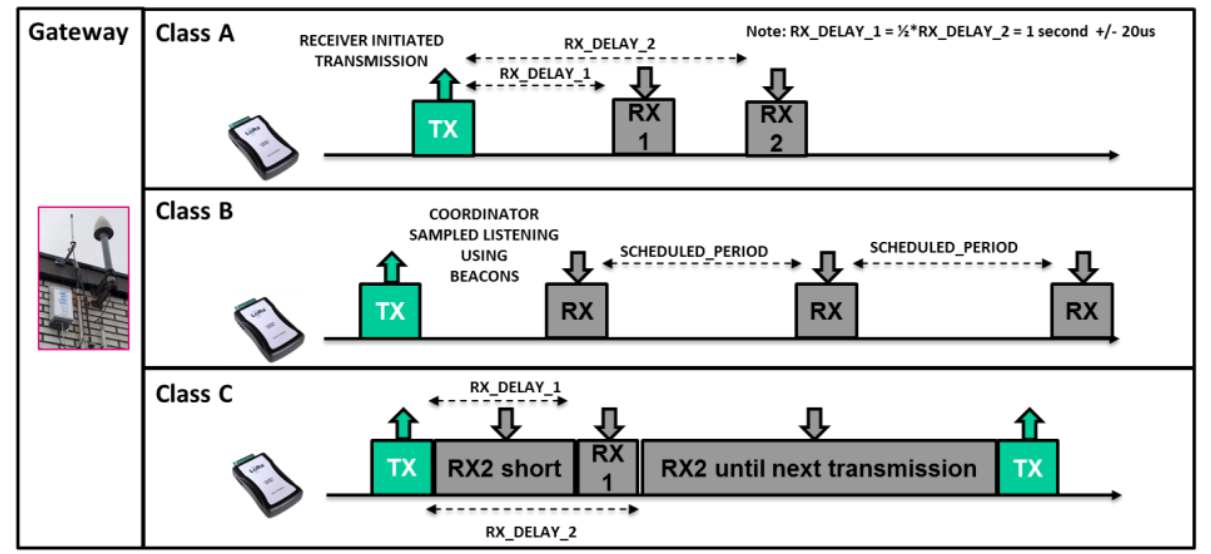

Figure 1. LoRaWAN supports 3 device classes. Class A - bi-directional end-devices (uplink transmissions are followed by two short downlink receive windows). Class B - bi-directional end-devices with scheduled receive slots (extra receive windows at scheduled times). Class $\mathrm{C}$ - always on devices.

Currently, the promising LoRaWAN technology is still in its early phase of adoption but the technology will definitely mature during the coming years, especially when adding improvements regarding scalability, QoS, cross-layer optimizations, improved MAC features, cross-network coordination, etc. 


\subsection{IEEE 802.11ah}

The new IEEE 802.11ah standard, marketed as Wi-Fi HaLow, is a wireless communication PHY and MAC layer protocol that adapts the IEEE 802.11ac for us in the unlicensed sub-GHz frequency bands [2] [3]. It supports a wide range of bandwidths ( 1 and $2 \mathrm{MHz}$ mandatory, and 4,8 and $16 \mathrm{Mhz}$ optional), modulation and coding schemes such as Binary Convolutional Codes (BCC) and Low-Density Parity-Check (LDPC) encoding, making it a highly flexible technology. This enables a theoretical throughput up to $347 \mathrm{Mbps}$ at short ranges, and $150 \mathrm{kbps}$ up to $1 \mathrm{~km} \mathrm{[3].}$

In order to support large numbers of stations, the standard extends the range of Association IDs (AIDs), and thus the number of associated stations, from 2048 up to 8192 per access point (AP), and organizes them hierarchically in a four level structure to improve station management scalability. The new fast association mechanism improves association speed of stations when many of them try to connect to the AP simultaneously. Finally, the restricted access window (RAW) mechanism reduces contention by clustering stations into RAW groups and slots, only allowing the stations in one slot to contend for the channel at any point in time (Figure 2). As such, it effectively combines the efficiency of CSMA-CA and the determinism of TDMA into a dynamically adaptable MAC scheduler. RAW has been shown to significantly reduce collision probability and interference in dense networks [4], resulting in a potential throughput increase of $50 \%$ or more.

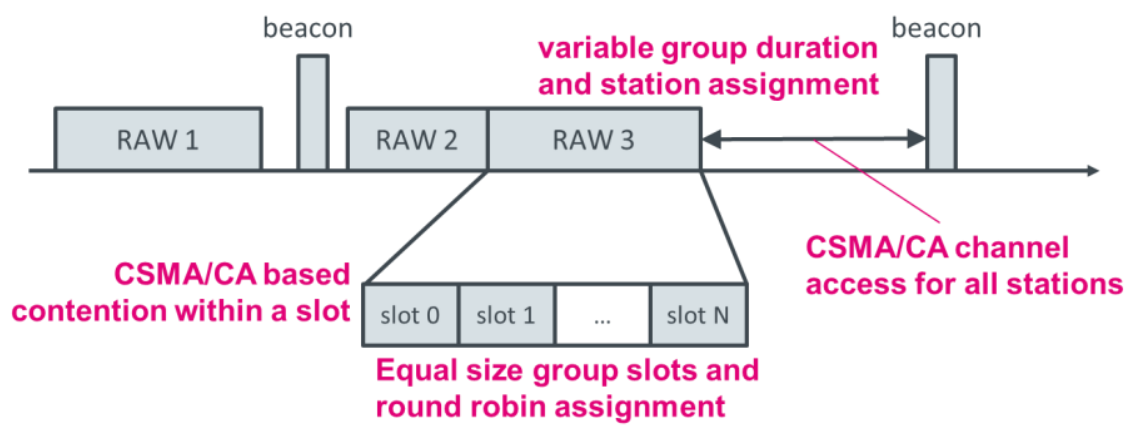

Figure 2. Conceptual representation of RAW groups. During each RAW group, medium access is restricted to a small sub-group of stations.

Traditional Wi-Fi stations need to wake up for every beacon frame, resulting in high power consumption. 802.11ah circumvents this by splitting the Target Information Map (TIM) into segments, each transmitted with another beacon. As such, stations only need to listen to beacons carrying the Delivery Traffic Indication Map (DTIM), which notifies the stations which beacon will carry their TIM segment. Subsequently, they only wake up to listen to that specific beacon, rather than all of them. The Target Wake Time (TWT) feature further reduces power consumption for stations transmitting data 
only occasionally, by letting them negotiate with the access point when they should wake up. TWT allows a sleep interval from seconds up to years.

In summary, 802.11ah is the most configurable technology of the LPWAN family, and can ride on the popularity of the Wi-Fi label. It is in a very early stage of adoption and open to a wide range of optimizations in terms of scalability, QoS support and energy efficiency.

\subsection{IEEE 802.15.4g}

IEEE 802.15.4g (marketed as WiSUN) is a recent PHY amendment to the IEEE 802.15.4 standard which is specifically tailored for long range communication [5]. It was originally designed for smart metering applications but is getting more and more popular in a wide range of long range IoT applications. It operates both in sub-GHz bands and the $2.4 \mathrm{GHz}$ bands. Depending on the PHY configuration, the data rates range from 40kbps up to 1000kbps. Moreover, as it supports PHY packet sizes of at least 1500 bytes, it is able to deliver IP packets without fragmentation.

As $802.15 .4 \mathrm{~g}$ only defines the PHY, typically IEEE $802.15 .4 \mathrm{e}$ is used as additional MAC layer. IEEE 802.15.4e was published in 2012 as an amendment to the MAC protocol defined by the IEEE 802.15.4 standard, mainly for improving the adoption of sensor node communication for industrial applications. One of the most promising new features is Time-Slotted Channel Hopping (TSCH), which is designed specifically to provide deterministic performance, ultra-low power consumption and network robustness, minimizing the impact of wireless unreliability. On top of IEEE 802.15.4e, 6TISCH is often used as an IPv6 adaptation layer [6]. The core mechanism of 6TISCH is to divide the time in slots of typically $10 \mathrm{~ms}$ and group them in frame slots. At each time slot several channels are available. As a result a Channel Distribution Usage (CDU) matrix is formed over multiple hops, where each cell is defined by a time slot and a channel offset. The Time Division Multiple Access (TDMA) nature of TSCH provides the capability of allocating a specific amount of bandwidth per node in preknown patterns. Additionally, channel hopping techniques address unreliability issues caused by factors such as with multipath fading and narrow-band external interference [7]. This combination of 6TISCH and IEEE 802.15.4g allows for an IPv6-enabled communication for long range IoT networks but the integration is still a study of research.

In summary, 802.15.4g is a long range extension of the popular 802.15.4 family of standards, arguably the most popular network solution for IoT to date. Its inherent multi-hop capabilities give it a unique edge to other LPWAN solutions.

\subsection{SigFox}

SigFox is a proprietary standard for long range IoT networks. SigFox is a narrowband (or ultra-narrowband) technology. It uses a standard radio transmission method called binary phase-shift keying (BPSK), and it takes very narrow chunks of spectrum $(100 \mathrm{~Hz}$, resulting in 8000 channels) and changes the phase of the carrier radio wave to encode the data. This allows the receiver to only listen in a tiny slice of spectrum which 
mitigates the effect of noise. This results in great sensitivity, which allows for longrange communication $(30-50 \mathrm{~km}$ in rural areas and $3-10 \mathrm{~km}$ in urban areas) at low bitrate (100bps), provided there is no interference.

These low SigFox bitrates mean that sending a SigFox packet requires a transmission time in the order of seconds, making it likely to collide with other technologies. Since SigFox does not employ any collision-avoidance techniques (no channel blacklistening, no listen before talk, etc.), and since such narrowband transmissions are the worst type of interferers for other systems, a single SigFox device can easily interfere with wideband sub-GHz technologies such as LoRa.

In contrast to the inexpensive radio of the end devices, the basestations use a sophisticated software-defined radio platform to simultaneously listen to all 8000 channels. To guarantee $99.9 \%$ reliability, each of the messages is sent 3 times on different frequencies to ensure it will correctly be received by at least one of the basestations in range. Considering the stringent transmit power limitations, and in Europe also the $1 \%$ duty cycle limitations, in practice per day up to 140 uplink messages of maximum 12 bytes can be sent per object and up to 4 downlink messages of 8 bytes can be received. A single SigFox-managed network is currently being rolled out worldwide, much like a cellular network.

In summary, the SigFox technology is suitable for very specific, very low data rate uplink IoT applications. Because of its closed nature it is difficult to innovate within SigFox for external researchers and companies. However, due to its popularity, it must be taken into account as a potentially harmful interferer for the other LPWAN technologies.

\section{Overall LPWAN management and coordination architecture}

As shown in the LPWAN overview section, one of the main limitations in wireless radio communications is the scarcity of available spectrum, which is the number one cause of network congestion and mutual interference among networks using overlapping frequency bands. This problem is especially prevalent in the unlicensed ISM bands. Recent wireless technologies targeting Internet of Things (IoT) applications, such as LoRa, 802.11ah, 802.15.4g, SigFox, DASH7 and many others, have moved away from the $2.4 \mathrm{GHz}$ band towards unlicensed spectrum in the sub-1GHz frequency range for several reasons. An important driver for this shift is the resulting longer range that enables the realization of a complete new range of low-energy IoT use cases. Moreover, the unlicensed sub-1GHz spectrum bands are considerably less utilized, circumventing the interference issues of the $2.4 \mathrm{GHz}$ band. However, as more and more IoT networks pop up that operate in sub-gigahertz frequencies and their usage becomes more popular, these bands will soon face similar interference issues. We envision an even grimmer future, as the amount of available spectrum is much smaller (i.e., an available bandwidth of $5 \mathrm{MHz}$ in the European $868 \mathrm{MHz}$ band compared to $80 \mathrm{MHz}$ in the $2.4 \mathrm{GHz}$ band) and the improved propagation range will cause interference at much larger scale. Clearly, if left unchecked the unlicensed sub-1GHz bands will soon be considerably congested and unusable. 
Although current solutions already include several coexistence and interference mitigation solutions, such as for example the use of channel hopping, this does not solve the problem, but merely postpones the inevitable overutilization of the entire spectrum band. At the core of this issue lies the fact that unlicensed networks are deployed, operated and managed in a completely uncoordinated manner, which leads to significant performance degradation.

In order to prevent this, there is a clear need for advanced and intelligent management, coordination and collaboration mechanisms that mitigate interference and improve coexistence among different networks (using the same and different technologies) operating in the unlicensed sub-1GHz frequency ranges. To this end, this paper proposes a research roadmap to realize an overall framework for the coordinated coexistence and management of wireless network technologies operating around $900 \mathrm{MHz}$ (e.g., 865-868 MHz in Europe and 902-928 $\mathrm{MHz}$ in the US), although many results and conclusions will also be applicable to other sub-GHz frequencies (e.g., $433 \mathrm{MHz}$ ).

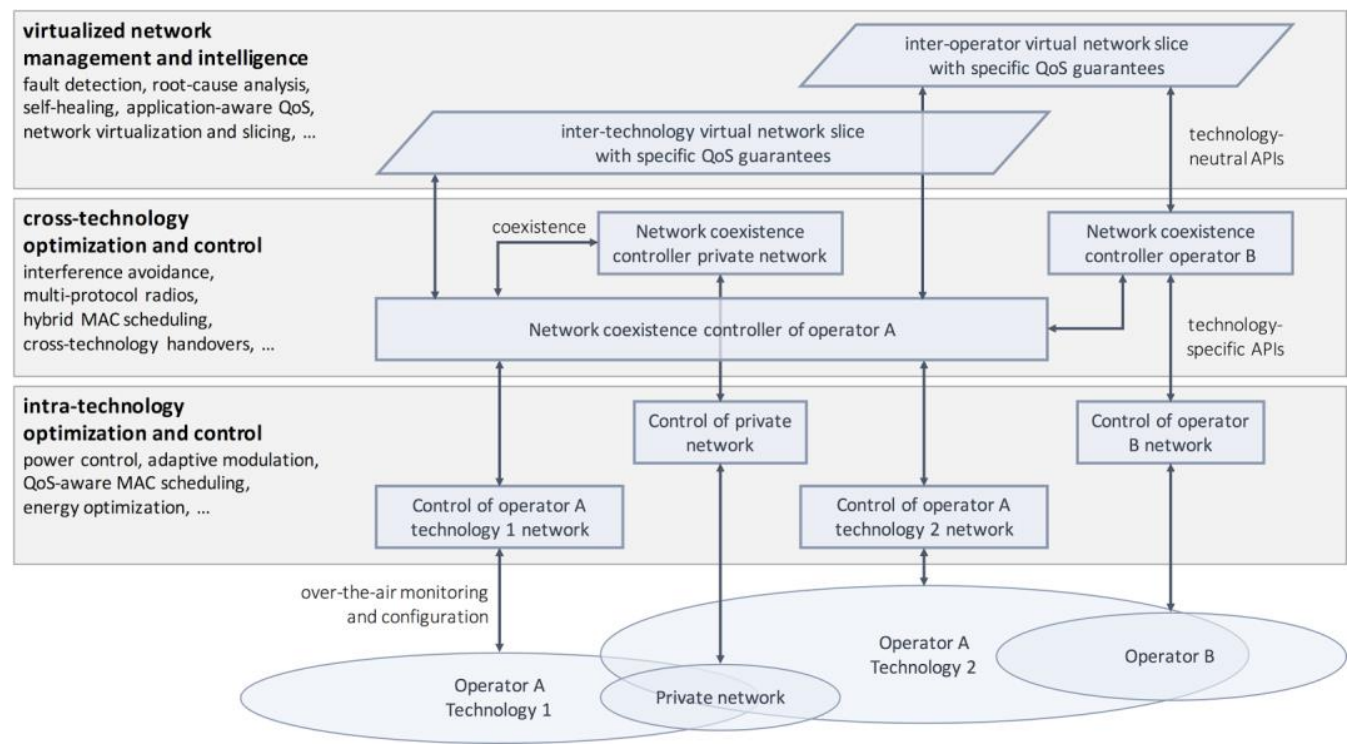

Figure 3. Overview of the three-layered LPWAN management and optimization framework, consisting of solutions for (i) intra-technology control, (ii) inter-technology coexistence and (iii) QoS-aware management of virtual LPWAN network slices.

As depicted in Figure 3, the functionality of the framework is spread over three-layers:

- Layer 1: intra-technology optimization and control. The bottom layer focuses on optimizing the different LPWAN technologies individually, in terms of scalability, Quality of Service (QoS) support and energy efficiency. 
Technology-specific as well as -agnostic solutions can be investigated. APIs of the developed solutions should be exposed to the higher layers of the framework, allowing them to be used as enablers for more elaborate control and management schemes that operate across networks.

- Layer 2: cross-technology optimization and control. The middle layer can leverage the lower layer technology-specific control and configuration options to increase coexistence among networks through interference avoidance techniques, cross-network negotiation and advanced coexistence coordination. Cross-technology operations will be further optimized through efficient multitechnology hardware sharing and inter-network routing solutions.

- Layer 3: virtualized network management and intelligence: The top layer should leverages the capabilities and solution developed at the two layers below in order to provide advanced management functionality through virtualization and slicing. The main feature of this layer is the ability to dynamically instantiate isolated virtual LPWAN network slices that deterministically satisfy specific QoS and application requirements. Slices transparently cross network and operator boundaries and scale based on evolving requirements.

To realize this vision, innovations at all 3 layers, ranging from the physical up to the application layer and potentially spanning the full chain of end devices, gateways and the cloud, are required thereby drastically improving the performance, scalability and manageability of long range wireless IoT technologies. The remainder of this paper outlines future research challenges at each of these layers, starting with those at intratechnology level.

\section{$4 \quad$ Technology innovation opportunities at intra-technology level}

This section discusses optimizations that improve the operations of individual LPWANs (i.e. within a single technology), without considering the impact of interfering networks (which will be discussed in Section 3 and 4 respectively).

\subsection{Challenges related to LPWAN scalability in dense network deployments}

Due to their large coverage domains, LPWAN technologies are expected to be used for large numbers of end devices (stations) that are deployed on a limited geographical area. To date, the problem of scalability in dense networks has mostly been studied in the context of cellular and Wi-Fi networks. Shin et al. studied Wi-Fi densenets and evaluated the three main approaches that can be applied to mitigate interference and therefore increase scalability in dense deployments [8]: (i) offloading to unlicensed LTE-U networks, (ii) more spectrally efficient modulation schemes (e.g., NOMA, SEFDM, OFDMA-VTS) and (iii) controlling interference levels by using 802.11ax features. However, these solutions cannot easily be adapted to sub-1GHz communications, due to their technology-specific nature, and high resource and energy requirements. Recent research performed has nevertheless shown that scalability in dense networks is also a significant bottleneck in LPWAN networks, such as LoRa and 
SigFox [9]. In the remainder of this section, we review state of the art techniques for improving efficiency specifically in dense LPWANs and provide future research directions to improve scalability.

\subsubsection{PHY level improvements}

Since the scalability of MAC protocols is inherently limited by the PHY layer time constraints (e.g. for carrier sense, for packet transmissions, etc.), PHY layer mechanisms will be explored to minimize the PHY network operation times. PHY layer improvements include (i) fast and low power synchronization for low duty cycles (allowing scalability by supporting smaller MAC slots), (ii) wideband spectrum sensing (allow the design of more scalable carrier sense solutions) and (iii) fast low power network joining procedures (to accommodate more sleeping devices to be connected to the same access point).

\subsubsection{MAC level improvements}

Improvements in scalability can be achieved at the MAC layer, by introducing appropriate scheduling in dense networks. Operation in the sub-GHz band either requires duty cycling (only transmit $0.1 / 1 / 10 \%$ of the time, depending on the frequency band used) or a MAC protocol implementing a Listen Before Talk with Adaptive Frequency Agility mechanism. LoRa and SigFox adopt a duty cycling mechanism: stations transmit whenever there is data to be sent, while adhering to the duty cycle limits. This lack of coordination regarding medium access will impact the scalability of the network when the number of stations grows. Today, LoRa and SigFox scalability can only be achieved by deploying more gateways and, for LoRa, by using an Adaptive Data Rate (ADR) mechanism to let devices communicate at a lower spreading factor in order to significantly reduce the airtime [1]. This only partially solves the scalability problem and, therefore, future work regarding the scalability of LoRaWAN networks must investigate ADR-aware MAC scheduling protocols to better coordinate access to the wireless medium. In addition, future optimizations include the investigation of the use of adaptive power control as a scalability feature in LPWAN networks. Concretely, adaptive power control can be used to dynamically adapt the range of transmissions based on the distance between sender and receiver. This allows the interference among stations to be greatly reduced, especially in densely deployed areas where a short transmission range is sufficient to reach the next hop.

Today, many existing LPWAN MAC scheduling protocols are based on CSMA-CA, which requires very limited coordination between the AP and stations, and is very bandwidth efficient. However, as the number of stations attempting to access the channel increases, so does the chance of collisions. This in turn increases the backoff timers and waiting times, causing highly degraded performance [10]. In contrast, TDMA-based MAC protocols avoid contention altogether. However, as the number of transmitting stations in the network grows, sending slot opportunities decrease, causing ever-growing latencies [11]. Time-synchronized channel hopping (TSCH), which is part of the 802.15.4e amendment to the IEEE 802.15.4 standard, increases the number of transmission opportunities by dividing both the frequency and time domains into slots [6], mostly using centralized schedulers. However, For large scale and dense multihop deployments, a centralized TDMA TSCH scheduler is infeasible as the overhead is 
too high [12]. Instead, decentralized schedulers such as proposed in [13] are being introduced but they again come with severe drops in throughput performance as the number of nodes grows, as sub-optimalities in the distributed schedule lead to cell (=slot+frequency) overlapping and therefore collisions. More research is needed to investigate the performance of such intelligent and automated scheduling mechanisms in the context of long-range IoT networks with only 1 to few hops and that can accommodate a variety of traffic patterns.

IEEE 802.11ah offers the Restricted Access Window (RAW) mechanism to increase scalability. Recent research has shown that, compared to the traditional 802.11 CSMACA scheduler, RAW-based scheduling can easily achieve a 50\% throughput increase in a 512 station network [14] [4]. The standard, however, only describes the RAW signaling and transmission protocol, and not the algorithm to configure the different RAW parameters (i.e., number of RAW groups and slots, how to split stations among groups, and duration of each group). Nevertheless, Tian et al. recently showed that the optimal RAW parameter values are highly dependent on a variety of network conditions, such as number of stations, traffic load and traffic patterns [4]. To date, only limited research has been performed on finding the optimal RAW parameters. In [15] a grouping method is proposed that exploits the random arbitration interface space number scheme and evaluate the average number of contending stations, throughput performance and energy-efficiency in saturation mode. However, they assume stations stay awake to listen to all beacons, negating the power-saving improvements of 802.11 ah and limiting applicability in power-constrained environments such as the IoT. [16] propose new holding schemes for the non-cross slot boundary case in the RAW mechanism, in which stations prevent their transmission from crossing the boundary of their allocated RAW slot, to improve saturated throughput and energy efficiency. [17] studies transmission latency and energy efficiency performance when RAW is applied to solve the hidden nodes problem for a large outdoor network. Finally, [18] propose an algorithm to determine the optimal size of RAW slots, considering the relationship between the estimated number of devices and the size of RAW in saturation mode. However, their approach only considers number of upload stations as a relevant input variable to determine the RAW parameters, while it is known that other aspects, such as traffic load and pattern, are equally important [4]. Moreover, they do not consider division of stations (i.e., each station randomly selects a RAW group) and are limited to scenarios with upload traffic only (e.g., sensor-based monitoring). Station grouping algorithms need to be developed that take into account not only the number of stations, but also their dynamic traffic patterns and intensities, as well as both upstream and downstream traffic [19].

Finally, it is clear that the benefits of these different MAC optimizations from different technologies are not yet well-compared, and optimizations from different technologies could easily be combined to create even more flexible and adaptive MAC protocols, e.g. by combining the TSCH channel hopping and grouping mechanisms into a single MAC protocol.

\subsubsection{Improvements through resource sharing}

A next set of scalability improvements can be expected through resource sharing and inter-LPWAN coordination. The LPWANs considered all operate in unlicensed 
bands, allowing anyone to operate its own LoRaWAN, 802.15.4 or HaLow network. Considering a single LPWAN technology, this will result in a multitude of co-located networks without any coordination and thus reduction in scalability due to interference. However, the presence of this entire infrastructure might actually be exploited in order to improve scalability. For instance, through virtualization (i.e. sharing the network infrastructure), LoRaWAN gateways owned by another operator might be reused, resulting in a denser network with more opportunities to use the ADR mechanism. Similarly, denser HaLoW networks enable higher bit rates and thus less airtime. Such resource sharing allows LPWAN networks to use each other's network infrastructure (gateways/APs), resulting in potential gains in scalability due to reduced airtime (shorter distance to infrastructure).

To realize resource sharing, multiple networks need to cooperate, requiring crosscoordination of scheduling to reduce interference between co-located single-technology LPWANs. So far, existing efforts in virtualizing wireless networks have focused on 3GPP LTE and IEEE 802.11 [20] [21] [22]. Research is needed to design virtualization solutions and management techniques for single-technology LPWAN networks and to investigate the gain in scalability that can be achieved by applying appropriate coordination mechanisms.

\subsection{Challenges to realize deterministic behaviour and QoS guarantees in LPWANs}

In addition to scalability challenges, the success of LPWANs for many application domains will depend on the capabilities of low-power wireless networks to satisfy the requirements of mission critical IoT services by providing deterministic guarantees in terms of QoS parameters (e.g., throughput, latency). Quality of Service (QoS) has traditionally been provided in wireless networks in two ways: (i) probabilistic QoS and (ii) deterministic QoS. The former increases the chance to successfully transmit data for high-priority traffic and is very popular in combination with CSMA-CA based MAC schedulers. The most common example is the 802.11e QoS standard for Wi-Fi that introduces enhanced distributed channel access (EDCA) and HCF Controlled Channel Access (HCCA). The former splits traffic into four classes and reduces the inter-packet waiting time, called arbitration inter-frame spacing (AIFS), for higher priority classes. This effectively increases throughput and reduces latency for such traffic. However, since channel access is still randomized to some extent, no real guarantees can be offered in terms of QoS. HCCA allows contention-free channel access, but is an optional feature with very little support in real hardware. Both EDCA and HCCA are also supported in 802.11ah. Deterministic QoS approaches, such as HCCA, reserve the wireless channel exclusively for one station at fixed intervals and are often used in combination with TDMA-based protocols. This takes away randomization, and increases the predictability of QoS parameters such as throughput and latency. Existing wireless technologies generally provide probabilistic QoS at best, which is incapable of offering the worst-case bounds required by many critical 
services, such as industrial IoT, healthcare and traffic safety applications. In order to achieve this challenges, several potential innovations are outlined in this section.

\subsubsection{Improvements related to channel hopping scheduling}

This approach is further extended in time-synchronized channel hopping (TSCH), which assigns slots to sender-receiver pairs in both the time and frequency domain. By assigning sufficient or sufficiently frequently slots, QoS requirements can be met. It is standardized as IEEE 802.15.4e and runs on top of the IEEE 802.15.4 physical layer protocol. The TSCH approach is very popular in critical IoT services, such as industrial applications, due to its predictability and deterministic nature. For example, in [23] it is shown that, by using TSCH, a deterministic guarantee can be provided on the bandwidth in a smart grid environment. However, as they only considered a smart grid environment, their results are not widely applicable to all possible IoT networks. At the heart of TSCH is the scheduler that decides which frequency-time slot(s) to assign and that can be either centralized or decentralized with each approach having its own advantages and disadvantages in terms of performance, scalability, etc. Equally or even more interesting in the light of QoS are the higher-layer control mechanisms to dynamically adapt the TSCH schedule to changing network and application requirements. For instance, [24] introduces an abstraction layer with virtual slots for decentralized scheduling that can accommodate different traffic types. Depending on the traffic type and topology information, a virtual slot can be mapped to one or more TSCH slots. The 6TiSCH working group [6] aims at integrating TSCH within the IoT protocol stack (CoAP - UDP - IPv6/6LoWPAN) and the dynamic allocation of cells to adapt to variations in throughput. This involves the definition of a new sublayer, 6top, allowing a management entity to control the TSCH schedule, collect connectivity information and monitor performance. It is important to note that existing scheduling methods do not consider the QoS requirements of stations and flows, but focus instead on global throughput maximization. In addition, it is currently unclear how effective channel hopping is in more limited spectrum bands with very few available channels.

\subsubsection{Improvements to slot scheduling}

Similarly to TSCH, the 802.11ah RAW mechanism can be adopted to provide probabilistic and deterministic QoS. By reducing the number of stations in a RAW slot, their chances of accessing the medium increase. By reducing the number of stations in a group, determinism for stations in that group can be improved. When a group consists of only a single station, its behaviour becomes fully deterministic in terms of throughput, latency and packet loss (barring outside interference). Increasing the slot size will increase throughput, while increasing the number of slots will additionally reduce latency. The RAW mechanism is highly flexible, allowing to provide both probabilistic and deterministic QoS to different stations at the same time. To date, no research exists that explores using RAW for QoS purposes. Both TSCH and RAW can only be used to provide differentiated service up to station and not flow level. However, since 802.11 ah also supports the EDCA mechanism, RAW can be combined with flowlevel differentiation. Determinism generally comes at the expense of latency efficiency, due to its TDMA-based nature. This is especially true for low-power communications. Improved latency can be achieved by providing low latency stations with more transmit 
opportunities, or combining deterministic with probabilistic QoS (e.g., integrating RAW and EDCA for 802.11ah). Also hybrid CSMA-TDMA scheduling can potentially provide deterministic low-latency QoS guarantees, with minimal energy increase.

\subsubsection{Automated derivation of application requirements}

Finally, several research opportunities exist regarding the integration with the higher layers of the IoT stack in order to take into account the application specifics and requirements. Research opportunities lie in the application-driven adaptation of the aforementioned mechanisms to provide application-driven or automated QoS configuration based on the application requirements. Applications must have a means to state their QoS requirements either (i) via well-defined APIs or (ii) management entities must become able to automatically derive such requirements. To realize the former, a light-weight signaling protocol (e.g. based on CoAP) could be derived to enable an application to explicitly share its QoS requirements with the network (relay nodes, gateways, etc.) via a well-defined API, for use by the scheduling algorithms. To realize the former, several research approaches exist. IoT applications have typical interaction patterns and higher-layer IoT protocols such as CoAP have their own specific mechanisms and timings regarding reliability, retransmission, etc. It should be possible to automatically collect and derive application requirements by inspecting the RESTful CoAP interactions being established and/or consulting directory services that provide insights in the application interactions (e.g. bindings between sensors and actuators, group communication manager, observe relationships for monitoring, etc.). Once these interactions are known, the corresponding attributes of the involved REST resources can be retrieved, allowing derivation of the required QoS based on the type and meaning of the resource. Both approaches will bridge the gap between the higher and lower layers and enable application-driven adaptation of the designed scheduling solutions.

Finally, and similarly to Section 4, improvements in deterministic communication are to be expected through inter-LPWAN coordination (with or without resource sharing through network virtualization), a currently unexplored research area.

\subsection{Challenges to improve energy efficiency in LPWANs}

Energy efficiency is crucial for LPWAN networks for the low-power operation of the end devices.

\subsubsection{Improvements at PHY layer}

For current sub-GHz transceiver designs, the PHY architecture and design are determining the power consumption. For receivers, an increase in sensitivity, bit rate or linearity typically leads to an increase of power consumption. Especially the receivers supporting a data rate higher than $1 \mathrm{Mbps}$ consume more than $5 \mathrm{~mW}$, whereas the sub$\mathrm{mW}$ and low-mW receivers support up to a few hundred kbps. Examples of low-power recent standard-compliant receivers are [25] [26], both having low power consumption, while supporting 3 different types of modulation at the cost of a lower, but still reasonable, sensitivity. 
For transmitters the power consumption is highly dependent on the output power. In high transmit power modes the Power Amplifier consumes more than 50\% of the total power budget. Theoretically FSK transmitters should be able to consume less power than phase modulation transmitters, since a non-linear (class C or D) PA could be used for constant envelope modulations such as GFSK. Trade-offs need to be made between low energy consumption [26], or increased bit rates such as [25] and [27]. Some reported low-power receivers and transmitters only implement frequency modulation. Since frequency modulation is a constant amplitude modulation scheme both the receiver and transmitter power consumptions can be decreased. In a receiver a hardlimiter can be used and in a transmitter a switching class PA can be used. Whereas OFDM typically does not provide for ultra-low power implementation due to the large peak to average ratio and resulting linearity demands on RF components. Significant efforts are still required to make tranceivers even more energy efficient, ideally up to the point that the lifetime of the battery becomes larger than the lifetime of the device itself.

In addition, for LPWAN devices that are in sleep modus for a long time, the network joining procedure (e.g. scanning for beacons) can consume a significant amount of energy and time, depending on the number of channels [28]. Dedicated PHY hardware potentially could accelerate clear channel assessment and network joining procedure time by wideband spectrum sensing techniques, listening to multiple channels and the same time, scanning for clear channels and/or beacons. Another option is to explore low power synchronization techniques, facilitating extreme low duty cycle and fast and efficient synchronization.

\subsubsection{Improvements at MAC layer}

Similar to QoS improvements, MAC scheduling algorithms for improved energy consumption can be improved. The MAC layer station grouping mechanism can serve, in addition to optimizing throughput and latency, as a mechanism to reduce energy consumption (as stations can sleep outside their group intervals).

\section{$5 \quad$ Technology innovation opportunities at cross-technology level}

In contrast to the previous section, which focuses on innovations within a single technology, this section describes innovation opportunities across multiple LPWAN technologies, mainly focusing on performance enhancements through interference mitigation and automated technology selection.

\subsection{Uncoordinated coexistence improvements for LPWAN technologies}

Significant research has been performed on this topic throughout the last decade, focusing mostly on the coexistence in the 2.4Ghz ISM band of IEEE 802.11 (Wi-Fi), IEEE 802.15.4 (ZigBee) and IEEE 802.15.1 (Bluetooth) [29], [30]. Measurement studies have shown mutual degradation of $802.11 \mathrm{~b}$ and 802.15 .4 , with throughput degradation for 802.11 of up to $80 \%$ [31] and Wi-Fi interfering with Bluetooth despite 
the use of Frequency Hopping Spread Spectrum (FHSS) [30]. As existing research is heavily focused on coexistence in the $2.4 \mathrm{GHz}$ band, little is known about the effects of coexisting wireless networks in Sub-1Ghz frequency bands. Nevertheless, similar effects are to be expected due to the plethora of LPWAN technologies operating in the same unlicensed band, especially since their low-power nature makes these networks very susceptible to interference from outside sources. An example waterfall plot of the full $868 \mathrm{MHz}$ spectrum of a SigFox base station in Paris is shown in Figure 4, showing that already now the frequency band can be extremely crowded at some locations. It is important to note the presence of a continuous interferer (left side of the figure) that is not EC certified and does not follow the EU duty cycle regulations, likely a device for e.g. communication between a crane operator and a construction team on the ground. This demonstrates that the ability to identify the presence of the different technologies that are utilizing overlapping radio bands is crucial.

As such, there is a need for further research into interference among sub-1Ghz wireless technologies. To this end, it is necessary to (i) be able to identify and correctly classify the presence of interfering technologies, (ii) combine interference information from different locations to obtain a wide-area view on the interference that is present and (iii) select the appropriate mitigation strategy.

\subsubsection{Challenges to identify and correctly classify the presence of interfering} technologies.

Past work in the $2.4 \mathrm{GHz}$ band has shown that this can be done effectively using dedicated hardware [32], which is not feasible for low-cost LPWAN end devices. Alternatively, by sampling link quality and/or received signal strength values in combination with processing it has been shown to be possible to identify Wi-Fi signals using commodity ZigBee nodes [33] [34], to identify ZigBee signals using commodity Wi-Fi cards [35] or to detect Wi-Fi idle intervals to which ZigBee frame lengths and transmission intervals can be adapted [36]. Other state of the art coexistence techniques utilize e.g. spectrum scanning for dynamic frequency allocations or utilize continuous frequency hopping to solve the interference problem [37] [38] [39] [40] [41]. The latter only offers limited opportunities for hopping due to the severe limitations in available spectrum (e.g., $5 \mathrm{MHz}$ available bandwidth in the $868 \mathrm{MHz}$ band in Europe). As such, research is needed to investigate to what extent spectrum sensing techniques using the available LPWAN chips or dedicated spectrum sensing devices can be used to identify interference levels and even identify the interfering technology. This includes the design of machine learning techniques to correctly identify the available spectrum and the types of LPWAN technologies that are currently present in an area based on the spectrum and network performance information (packet loss rates, typical sizes of error bursts, etc.) from these sources.

\subsubsection{Combining interference information from different locations to obtain a wide-area} view

Most existing technology recognition approaches assume the same interference is present in the monitored area. However, due to the large coverage area of LPWAN technologies, a single spectrum sensing device is not sufficient to obtain accurate insights in the presence of LPWAN technologies over a wide area. To this end, there is 
a need for novel distributed spectrum sensing techniques that utilize information from multiple, heterogeneous sub-GHz radio sources. These heterogeneous sources can include SDR radio platforms, as well as multiple off-the-shelf LPWAN radios of different technologies spread out over an area. Each of these radios offers different radio frequency front-end flexibility, sensing speed and accuracy and varies in the way the samples are processed and stored, each giving different insights and accuracies. The outcome of these integrated insights should result in an area-wide overview of available spectrum and the expected type and frequencies of interfering technologies.

\subsubsection{Selecting the appropriate mitigation strategy}

Finally, once the interference present in the environment is correctly mapped, appropriate interference mitigation techniques can be applied. Since frequency hopping opportunities in these bands are often limited or non-existent for many sub-GHz technologies that use wider bands due to the severe limitations in available spectrum (e.g., $5 \mathrm{MHz}$ available bandwidth in the $868 \mathrm{Mhz}$ band in Europe), it is likely that these innovations should mainly focus on PHY/MAC reconfiguration mechanisms designed to improve coexistence. Examples include automatic selection of more robust coding schemes, limiting packet sizes, increasing error correction codes, or simply defining an ad-hoc and very granular cross-technology TDMA scheme, etc.

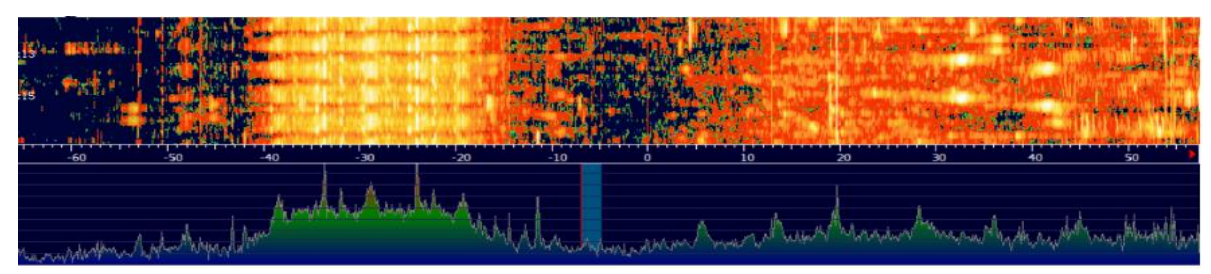

Figure 4. Top: waterfall plot of the $868 \mathrm{MHz}$ spectrum from a SigFox base station. The intensity of the medium use is represented using a colour code (lighter colours correspond to higher signal strengths), where the $X$-axis represents the $868 \mathrm{MHz}$ spectrum and the $Y$ axis the time. Bottom: instantaneous distribution of the current signal strengths over the full $868 \mathrm{MHz}$ ISM spectrum. Depending on the location of a base station, a significant amount of the spectrum is already occupied.

\subsection{Coordinated coexistence of LPWAN technologies}

The uncoordinated coexistence improvements are only viable up to a certain extent, as they are not effective when the spectrum is strongly overused and when the number of non-overlapping channels is limited. Further, considering the fact that MAC solutions will be moving towards efficient, adjustable TDMA schemes, it becomes much more useful to exchange and coordinate MAC schedules, rather than spending time for detecting interference or other ongoing transmissions. Therefore, for more scalable coexistence solutions, devices should be able to negotiate between each other.

In contrast to uncoordinated coexistence techniques (CSMA/CA, channel hopping, etc.), coordinated coexistence approaches allow improved coexistence through negotiation about optimal PHY and MAC settings between different wireless technologies. E.g. in [42], a centralized controller is used to assign time slots of a TDMA protocol for $\mathrm{WiFi}$ and ZigBee devices, avoiding interference between them. 
However, due to the strict timing requirements, the approach is only available for radio chips that have a direct communication approach (e.g. the radios are connected to the same devices, or a wired backbone between them exists). In addition, the solution is specifically designed for WiFi and ZigBee, and as such cannot directly be applied for coexistence of sub-GHz technologies that are not connected to the same centralized controller. Due to the long range of LPWAN technologies, LPWAN coexistence solutions should be suitable also for non co-located devices. As such, in contrast to recent approaches such as [42] that requires direct (serial line) communication between the radios from different technologies, negotiations between different radio technologies from different owners should be possible.

Future research could focus on the design of novel cross-technology communication approaches for communicating between LPWAN devices, suitable for communication between devices from different technologies. Two example mechanisms are the following. First of all, a technology-independent communication mechanism (taking a similar approach as in [43]) that generates technology-agnostic signal patterns and properties to signal information between devices can be designed. This technologyagnostic communication channel can be used to exchange QoS requirements, but also for cross-technology synchronization between end devices from different sub-GHz technologies, allowing the design of cross-technology TDMA protocols between heterogeneous LPWAN technologies. Alternatively, it could be interesting to investigate how different co-located and interfering LPWANs can exchange and agree upon MAC schedules (frequencies used, division of time, etc.) through communication over the LPWAN backbone network (i.e. between gateways, network servers, etc.). Crucial here will be accurate synchronization between the different actors, as inaccuracies will either lead to periods with interference or underutilization of the medium.

\subsection{Challenges related to technology handovers and multi-technology devices}

As the LPWAN technologies are currently quite diverse in supported bitrate, energy, etc. they are typically strongly linked with the application they need to support. As such, they are not strong in providing changing application requirements in terms of throughput, latency, etc. To support devices with dynamic application requirements (e.g. for logistics applications), multi-technology devices are required. In addition, although the ranges LPWANs support are significant, there is still no global coverage of the same LPWAN technology and in many cases, deep indoor coverage is still lacking. As such, the availability of multiple technologies is also important for mobile nodes that currently suffer from frequent connectivity outages. To remedy both scenarios, from the device point of view a global LPWAN network can be formed by grouping the different technologies (IEEE 802.11ah, IEEE 802.15.4g and LoRaWAN) together into one wide area network. This means the ability to seamlessly switch from one technology to the other and to form even multi-hop LPWAN networks that span multiple LPWAN technologies. To realize this goal, several research challenges need to be solved. First, it should be investigated how traditional routing algorithms can be integrated within the LPWAN standard to both automatically find the best performing paths that lead to a global coverage and delivering packets with minimal latency and 
energy consumption. Second, new handover mechanisms should be designed that are able to switch between technologies in a way that is transparent both to the application at the end node and the central (cloud) site. As LPWAN technologies typically do not assume a continuous stream of data, the focus is more on fast handovers, with limited signaling and energy consumption and less on the preservation of sessions. Third, multihop and multi-technology networks should cope with common optimization challenges such as the load-balancing between technologies, addressing temporal outages of a specific technology through handovers and incorporating the concept of technology selection in which the radio switches to a different less interfered gateway. Finally, to realize this, there is a need for a virtualized LPWAN interface, which provides an additional abstraction on top of multiple LPWAN technologies (both in gateways and end nodes). This virtualized radio is the only interface that is provided to the application: it will be responsible for transparently mapping the traffic onto the physical radios (both LPWAN and other wireless technologies) and has the ability to do handovers or load balancing between the radios.

\section{Technology innovation opportunities for LPWAN network management, LPWAN virtualization and optimization}

The previous subsections introduced several innovation paths to improve single and multi-technology LPWAN performance and coexistence involving increased reconfigurability, inter-LPWAN coordination, resource sharing, cross-layer information exchange, etc. Such an increase in flexibility and configuration capabilities or the introduction of resource sharing and virtualization requires the use of appropriate virtualization techniques, management mechanisms and APIs in order to intelligently orchestrate and steer the network configurations. Next to this, additional intelligence can be introduced to properly manage the devices and the traffic flows to and from the LPWAN networks. This section discusses state of the art approaches and future research directions for the management of LPWAN networks, including (i) virtualized LPWAN infrastructures, (ii) optimization methods to automatically manage and reconfigure (virtualized) LPWAN, (iii) enablers for device reconfigurations and software updates.

\subsection{Challenges related to virtualization of LPWANs}

Virtualization can aid network management in hiding the inherent complexity of multiple complementary radios by providing a single virtualized interface, especially when designing management solutions for multi-technology LWPANs. For example, a single operator might deploy a multi-technology LPWAN network that must be managed in a uniform way or end users having multi-technology devices in the field want to perceive them as a single logical network. Existing efforts in virtualizing wireless networks have focused on 3GPP LTE and IEEE 802.11 [20] [21] [22]. These efforts aim at slicing the wireless network, meaning that the infrastructural resources can be shared and isolated, and configuration and management can be simplified. Each 
slice supports a number of flows, possibly each with its own QoS requirements, and consists of a subset of network resources. It also support slides used by different operators offering their own services. Related concepts are Wireless Network Virtualization where the same radio resources are shared by different virtual networks (relevant for LoRaWAN, HaLoW and 802.15.4g to optimize operation in unlicensed band), Software Defined Networking to decoupling the data and control plane (already applied partly by LoRaWAN through the Network Server, but relevant for multi-AP HaloW and 802.15.4g deployments and remote MAC control as well) and Network Function Virtualization to decouple functions from the physical network equipment. Besides heterogeneous service differentiation for QoS control, virtualization also helps to manage wireless networks with heterogeneous underlying technologies, which perfectly applies to multi-technology LPWANs as no single technology can meet all IoT use cases requirements. In addition to the design of these virtualization approaches, a major challenge will consists in the combination of these LPWAN mechanisms with existing solutions for virtualization on top of wired networks, in order to ensure optimal transport on the end-to-end path.

To date, the ability to improve user mobility, load balancing, QoS control and reliability by using virtualized wireless networks is largely unexplored. Virtual network embedding (VNE) and programmable access points have been studied to allow for flexible network management in multi-provider scenarios [44] [45], but do not provide sufficient QoS and SLA guarantees in dynamic scenarios. For 802.11ah, the challenges related to the randomness and distributed aspects of the access control can be tackled by traffic shaping and EDCA control for (downlink and uplink) slicing, as suggested by the most comprehensive works on 802.11, such as [46]. Further, when involving LPWAN end devices, appropriate signaling mechanisms need to be considered, given the constraints in bandwidth and device capabilities. [47] presents a constrained signaling approach based on CoAP for operating such networks and minimizing the amount and size of messages.

\subsection{Challenges related to network optimization}

In order to reduce the load on the LPWAN end devices and to control the traffic to and from the LPWAN networks, additional intelligence can be deployed at the border of the LPWAN network [48]. This approach is often referred to as "edge" or "fog" computing [49] [50], and can be used to migrate otherwise complex to implement functionality away from the constrained LPWAN end devices (called Sensor Function Virtualization, see [48]) or to avoid entering unnecessary traffic into the LPWAN. Additionally, it can be used to control the way how traffic is injected into the network, enabling alignment with underlying MAC schedules. By keeping the processing at the edge of the network (rather than in a remote cloud), the optimizations can take into account network characteristics such as wake-up times, thereby enable additional LPWAN optimizations beyond current state of the art.

Due to the remote accessibility of many LPWAN end devices and the increasing configuration complexity, these network configurations need to be optimized in an automated way without human interactions, e.g. using machine learning approaches. As 
LPWAN networks and the involved end devices are typically constrained, it is interesting to introduce intelligence at the border of the network in order to reduce or control the load to and from the network or move away functions from the constrained LPWAN devices. For example, [51] proposes a neural network based mechanism for dynamic channel selection in an IEEE 802.11 network. The neural network is trained to identify how environmental measurements and the status of the network affect the performance experienced on different channels. In [52] an intelligent communication system is proposed called COgnition-BAsedNETworkS (COBANETS) for reconfiguration of the network protocol stack and flexible network management. A recent overview of existing machine learning solutions for wireless networks is given in [53]. However, currently there is a clear lack of self-learning systems for $\mathrm{sub-GHz}$ LPWAN technologies. In addition, the existing self-learning solutions are not designed to cope with network dynamics (such as changing topologies or node densities), changing application requirements or changing environmental conditions (such as varying interference levels).

The mentioned systems all consider simple, static systems with only a few parameters that can be optimized (e.g. typically 5 configuration parameters or less). To be useful for a wide range of conditions, a larger design space should be considered, including parameters from multiple protocol layers (PHY, MAC, etc.) as well as parameters that represent dynamic network aspects. However, considering a larger design space significantly impacts the time necessary to create a system model (e.g. the exploration phase). For speeding up the convergence of these complex systems, design of experiment methods \& dimensionality reduction techniques have been used to quickly identify the parameters with largest influence on the system performance, as well as the two-way interactions between these parameters. An example is given in [54], where a locating array is used to identify the 5 most influential parameters out of an initial design space of 24 parameters. As such, by applying dimensionality reduction techniques before model creation, the exploration time can significantly be reduced. However, none of these techniques have been applied to dynamic LPWAN systems, and as such an analysis on how inherent system noise and variability impacts the accuracy of the dimensionality techniques and model creation is missing.

Finally, in addition to the lack of dynamic self-learning solutions, most LPWAN devices will be too constrained to implement self-learning protocols. An alternative approach is the use of an online cloud repository, in which system models are stored. These system models can be created using e.g. supervised machine learning models and can be contacted whenever end devices encounter a failure or non-optimal configuration. As such, the models represent the network performance (performance statistics gathered from the hardware/drivers/MAC layer), system failure states (e.g. link failure) and the corresponding root cause of these failures (misconfiguration, too large distance, external interference, etc.). Newly deployed systems can access this repository to download previous system models from similar environments and network conditions. This way, newly deployed systems, or systems for which environmental conditions have changed, can reuse existing models to avoid a lengthy exploration phase to adapt to the new conditions. In case no existing model is present in the cloud, a new model is created by the devices and made accessible for future networks. This way, learning occurs in the cloud, and constantly triggers improvements of all connected 
networks, allowing the intelligence framework to derive lessons learned and reasons for disconnections/deauthentications/failed transmissions based upon statistics gathered from the hardware/drivers/MAC layer. To realize such a cloud based repository, innovative solutions are required related to the following topics. (i) The design and evaluation different representation methods and supervised learning techniques for the creation of failure and performance models. (ii) An analysis on how the complexity of these models can be reduced, e.g. through dimensionality reduction techniques such as Locating Array approaches [54]. (iii) Analysis approaches related to how the LPWAN inherent system noise and variability impacts the accuracy of these models (before and after dimensionality techniques have been applied). (iv) Creation of an automatically generated online repository with occurring problems and their solutions. (v) Identification of which network and system features are best suited for selecting the most relevant models for newly deployed systems from the repository.

\subsection{Challenges related to device reconfiguration and software updates}

Many LPWAN use cases involve end devices having a targeted lifetime of 10+ years. Therefore, they do not only need to support reconfigurations, but they will also need to be wireless upgradable and reconfigurable to (i) support evolving wireless standards, (ii) perform bug fixes and (iii) support advanced reconfigurations, such as switching to a new MAC protocol for improving technology coexistence. As such, there is a need for efficient and reliable reconfiguration, including software updates, of potentially large groups of LPWAN devices. Due to the resource constrained nature of these (mostly) embedded devices, wireless upgrades should not interrupt the run-time operation of the devices and should be very efficient in terms of code update size.

There are several individual works that focused on this issue in for embedded devices [55] [56]. In these publications, the binary is no longer a single monolithic code block, but is instead a set of modular components that can individually be modified and/or replaced. Unfortunately, most current code update solutions are either limited to application level, or are too large to be suitable for low-data rate LPWAN devices. In addition, when a module that relies upon by another module is to be stopped, then the latter module needs to be stopped as well before being able to perform the update. It should be possible to update individual parts of the LPWAN protocol stack of embedded end devices, from the application layer down to the MAC layer. For the latter, a proper design of the MAC layer architecture has to be done in order to support on demand updates of the MAC protocol, allowing devices to reconfigure their MAC behavior after deployment. This is not possible in current MAC protocol architectures for embedded devices, which typically include hardware specific code and as such cannot be modified after deployment. As such, none of currently existing solutions offer runtime updates of code that directly interacts with hardware, such as MAC protocols.

Recently, the feasibility of code updates of components of both the network layers through the use of a dynamic component object model (COM) was demonstrated [57]. However, the architecture cannot yet be used for over-the-air updates of MAC protocols. Also, decision logic capable of deciding which configurations and protocols are optimal is still missing, e.g. machine learning optimizations that are capable of selecting and configuring the network protocols (including MAC) based on current 
environmental conditions (device density, interference levels, etc.) as well as the application requirements.

Finally, appropriate management tools and protocols are needed to reliably perform reconfigurations, status monitoring, diagnosis or software updates across large groups of devices. For this, group communication solutions based on standardized IoT protocols that are optimized for constrained devices might be used, as presented in [58] [59], but the feasibility of such solutions has not been evaluated over LPWAN networks.

\section{Conclusions}

Low Power Wide Area Networks or LPWANs provide significant improvements in terms of power consumption, coverage, deployment cost and pricing over cellular and other M2M connectivity technologies, and as a result strong adoption of unlicensed LPWAN solutions is expected in the coming years. As such, the forecasted numbers, growth margins, potential revenues and variety of IoT application domains that can be reached through LPWAN technologies in unlicensed spectrum are huge. However, currently several competing technologies are available, each with limited QoS support. Due to the lack of inter-technology coordination, and the strong potential of future performance degradation due to large-scale interference, sub-GHz networks might become a victim of their own success and become unusable in the future.

To remedy this, this paper proposes a conceptual coordination framework to uniformly manage an ecosystem of coexisting wireless sub-1GHz LPWANs. The framework greatly simplifies IoT service instantiation, as well as network operations by transparently initializing and managing virtual LPWAN slices that provide deterministic QoS guarantees on top of a set of highly heterogeneous networks, with varying features and capabilities. It achieves this through automatically and dynamically configuring a set of underlying technology-specific and -agnostic functionalities, which enable interference avoidance, scalability, QoS provisioning and energy efficiency. To realize this goal, multiple research innovations are required. As such, the paper outlines a series of innovation outcomes positioned at the three defined layers of the paper concept.

(i) At the level of intra-technology optimization and control, the paper describes current approaches and future research directions to improve the scalability (in terms of network density), to provide deterministic end-to-end QoS provisioning and to improve energy efficiency within different LPWAN technologies. (ii) At the level of intra-technology optimization and control, the paper describes current approaches and future research directions to detect and avoid interference, as well as to support multitechnology devices. (iii) Finally, technology agnostic solutions for virtualized LPWAN network management and optimization are proposed, such as the definition 
of light-weight virtualization APIs, a cloud repository suitable for optimization of newly deployed LPWAN networks, and fully reliable over-the-air, reconfigurations and partial software updates of large groups of devices.

A significant amount of research will be needed to realize all of these research innovations, but the authors are convinced that all of these research challenges are (i) useful even on their own and (ii) are necessary to successfully exploit all the capabilities of future LPWAN networks. As such, the open research trends identified in this work can pave the road towards realizing diverse and demanding IoT applications that benefit from future LPWAN technologies.

\section{Acknowledgements}

This work was carried out in the context of following projects. IDEAL-IoT (Intelligent DEnse And Longe range IoT networks) is an SBO project funded by the Fund for Scientific Research-Flanders (FWO-V) under grant agreement \#S004017N. 'Processing visual sensor data in low-power wide area networks' is a project funded by the Fund for Scientific Research-Flanders (FWO-V) under grant agreement \#G084177N". The UGent GOA "Disposable and biodegradable wireless networks for extreme conditions" project. The H2020 eWINE project under grant agreement number 688116.

\section{References}

[1] "LoRaWAN: What is it? - A technical overview of LoRa and LoRaWAN", A technical overview of LoRa and LoRaWAN, LoRa Alliance Technical Marketing Workgroup 1.0, November 2015.

[2] WG802.11 - Wireless LAN Working Group, "802.11ah-2016 - IEEE Approved Draft Standard for Information Technology", https://standards.ieee.org/findstds/standard/802.11ah-2016.html, February 2017 release.

[3] M. Park, "IEEE 802.11ah: sub-1-GHz license-exempt operation for the internet of things," IEEE Communication Magazine., vol. 53, no. 9, pp. 145-151, Sep. 2015.

[4] L. Tian, J. Famaey, L. Latré, "Evaluation of the IEEE 802.11ah Restricted Access Window Mechanism for dense IoT networks," IEEE International Symposium on a World of Wireless, Mobile and Multimedia Networks (WoWMoM), 2016.

[5] K.H Chang and B. Mason, "The IEEE 802.15.4g standard for smart metering utility networks," Smart Grid Communications, 2012 IEEE Third International Conference on, Tainan, 2012, pp. 476-480. 
[6] D. Dujovne, T. Watteyne, X. Vilajosana and P. Thubert, "6TiSCH: deterministic IP-enabled industrial internet (of things)", in IEEE Communications Magazine, vol. 52, no. 12, pp. 36-41, 2014.

[7] T. Watteyne, A. Mehta, and K. Pister. "Reliability through frequency diversity: why channel hopping makes sense". 6th ACM symposium on Performance evaluation of wireless ad hoc, sensor, and ubiquitous networks, okt. 2009.

[8] K. Shin, I. Park, J. Hong, D. Har, and D. Cho, "Per-node throughput enhancement in Wi-Fi densenets,” IEEE Commun. Mag., vol. 53, no. 1, pp. 118-125, Jan. 2015.

[9] M. Weyn, "Low Power Wide Area Networks", Available online: https://sites.google.com/a/wesdec.be/mweyn/lpwan, 2016, Last accessed: 18/05/2016.

[10] G. Daneels, J. Famaey, S. Bohez, P. Simoens, S. Latré, "Upstream content scheduling in Wi-Fi DenseNets during large-scale events", IEEE Globecom Workshop on Enabling Technologies in Future Wireless Local Area Networks (ETFWLAN), 2015.

[11] S. Zats, R. Su, T. Watteyne, and K. S. J. Pister, "Scalability of Time Synchronized wireless sensor networking," in 37th Annual Conference of the IEEE Industrial Electronics Society, 2011, pp. 3011-3016..

[12] M. R. Palattella, N. Accettura, M. Dohler, L. A. Grieco and G. Bog- gia, ”Traffic Aware Scheduling Algorithm for reliable low-power multi- hop IEEE 802.15.4e networks," Personal Indoor and Mobile Radio Communications, 2012.

[13] D. Dujovne et al., "6TiSCH On-the-Fly Scheduling," Internet-Draft [work-inprogress], IETF Std., Rev. draft-dujovne-6tisch-on-the-fly-02, 14 Feb. 2014.

[14] L. Tian, L. Latré, J. Famaey, "Implementation and validation of an IEEE 802.11ah module for NS-3," Workshop on ns-3 (WNS3), 2016.

[15] K. Ogawa, M. Morikura, K. Yamamoto, and T. Sugihara, "IEEE 802.11ah Based M2M Networks Employing Virtual Grouping and Power Saving Methods," IEICE TRANSACTIONS on Communications, vol. 96, no. 12, pp. 2976-2985, 2013.

[16] M. Qutab-ud-din, A. Hazmi, B. Badihi, A. Larmo, J. Torsner, and M. Valkama, "Performance analysis of IoT-enabling IEEE 802.11ah technology and its RAW mechanism with non-cross slot boundary holding schemes”, IEEE 16th International Symposium on A World of Wireless, Mobile and Multimedia Networks (WoWMoM), 2015.

[17] M. Park, "IEEE 802.11ah: Energy efficient MAC protocols for long range wireless LAN," IEEE International Conference on Communications (ICC), pp. 2388-2393, 2014. 
[18] C. W. Park, D. Hwang, and T.-J. Lee, "Enhancement of IEEE 802.11ah MAC for M2M Communications”, IEEE Commun. Lett., vol. 18, no. 7, pp. 1151-1154, Jul. 2014.

[19] Lei, X. \& Rhee, S.H. Wireless Personal Communications (2017) 93: 933. doi:10.1007/s11277-017-3947-3, 2017.

[20] C. Liang and F. Yu, "Wireless network virtualization: A survey, some research issues and challenges”, Communications Surveys Tutorials, IEEE, vol. 17, no. 1, pp. 358-380, 2015..

[21] F. Granelli, A. Gebremariam, M. Usman, F. Cugini, V. Stamati, M. Alitska, P. Chatzimisios, "Software defined and virtualized wireless access in future wireless networks: scenarios and standards", IEEE Communications Magazine, vol. 53, no. 6, 2015.

[22] M. Yang, Y. Jin, L. Zeng, X. Wu, A. Vasilakos, "Software-Defined and Virtualized Future Mobile and Wireless Networks: A Survey", Mobile Netw Appl (2015) 20:4-18, 2015.

[23] Paventhan, A., Krishna, H., Pahuja, N., Khan, M.F. and Jain, A., 2015, December. "Experimental evaluation of IETF 6TiSCH in the context of Smart Grid". In Internet of Things (WF-IoT), IEEE 2nd World Forum on (pp. 530-535), 2015.

[24] Duquennoy, S., Al Nahas, B., Landsiedel, O., Watteyne, T., Orchestra, "Robust Mesh Networks Through Autonomously Scheduled TSCH", In Proc. of the 13th ACM Conference on Embedded Networked Sensor Systems, pp. 337-350, 2015.

[25] Y.-H. Liu, X. Huang, M. Vidojkovic, A. Ba, P. Harpe, G. Dolmans and H. d. Groot, "A $1.9 \mathrm{~nJ} / \mathrm{b} \quad 2.4 \mathrm{GHz}$ Multistandard (Bluetooth Low Energy/Zigbee/IEEE802.15.6) Transceiver for Personal/Body-Area Networks," in ISSCC 2013, 2013.

[26] A. C. W. Wong, M. Dawkins, G. Devita, N. Kasparidis, A. Katsiamis, O. King, F. Lauria, J. Schiff and A. J. Burdett, "A 1 v $5 \mathrm{~mA}$ multimode IEEE 802.15.6/bluetooth low-energy WBAN transceiver for biotelemetry applications," Solid-State Circuits Journal, Volume: 48 Issue: 1, December 2012.

[27] P. D. Fabbro et al., "A 0.8V 2.4GHz 1Mb/s GFSK RF transceiver with on-chip DC-DC converter in a standard $0.18 \mu \mathrm{m}$ CMOS technology", Proceedings of ESSCIRC, 14-16 Sept. 2010, DOI: 10.1109/ESSCIRC.2010.5619742.

[28] Das, Kallol and Mathews, Emi and Zand, Pouria and Sanchez Ramirez, Andrea and Havinga, Paul, "Efficient I/O joining and reliable data publication in energy harvested ISA100.11a network",, IEEE International Conference on Industrial Technology (ICIT), 2015. 
[29] D. Yang, Y. Xu, and M. Gidlund, "Wireless coexistence between IEEE 802.11and IEEE 802.15.4-based networks: A survey", International Journal of Distributed Sensor Networks, vol. 2011, 2011.

[30] R. Challoo, A. Oladeinde, N. Yilmazer, S. Ozcelik, and L. Challoo, "An Overview and Assessment of Wireless Technologies and Coexistence of ZigBee, Bluetooth and Wi-Fi Devices," Procedia Comput. Sci., vol. 12, pp. 386-391, 2012.

[31] S. Pollin, Ian Tan, Bill Hodge, Ahmad Bahai, "Harmful Coexistence Between 802.15.4 and 802.11: A Measurement-based Study", Cognitive Radio Oriented Wireless Networks and Communications, 2008.

[32] Lakshminarayanan K, Sapra S, Seshan S, Steenkiste P, "Rfdump: An architecture for monitoring the wireless ether", In Proceedings of the 5th ACM International Conference on Emerging Networking Experiments and Technologies (CoNEXT), 2009.

[33] Hermans F, Larzon L-A, Rensfelt O, Gunningberg P, "A lightweight approach to online detection and classification of interference in 802.15.4-based sensor networks"', ACM SIGBED Review - Special Issue on the 3rd International Workshop on Networks of Cooperating Objects (CONET), 2012.

[34] Zhou R, Xiong Y, Xing G, Sun L, Ma J, "Zifi: Wireless LAN discovery via ZigBee interference signatures", In Proceedings of the Sixteenth Annual International Conference on Mobile Computing and Networking. MobiCom ' 10 . ACM, New York; pp 49-60, 2010.

[35] Rayanchu S, Patro A, Banerjee S: Airshark: Detecting non-WiFi RF devices using commodity WiFi hardware. In Proceedings of the ACM SIGCOMM Conference on Internet Measurement Conference. IMC ‘11. ACM, New York; pp137-154, 2011.

[36] Huang J, Xing G, Zhou G, Zhou R, "Beyond co-existence: Exploiting WiFi white space for Zigbee performance assurance", 18th IEEE International Conference On Network Protocols, pp 305-314., 2010.

[37] Q. Javed and R. Prakash, "CHAMELEON: A Framework for Coexistence of Wireless Technologies in an Unlicensed Band,” Wirel. Pers. Commun., vol. 77, no. 1, pp. 777-808, 2013.

[38] L. Tytgat, O. Yaron, S. Pollin, I. Moerman, and P. Demeester, "Analysis and Experimental Verification of Frequency-Based Interference Avoidance Mechanisms in IEEE 802.15.4," IEEE/ACM Trans. Netw., vol. 23, no. 2, pp. 369-382, Apr. 2015. 
[39] Correia2015, L. H. A. Correia, T.-D. Tran, V. N. S. S. Pereira, J. C. Giacomin, and J. M. Sá Silva, "DynMAC: A resistant MAC protocol to coexistence in wireless sensor networks,” Comput. Networks, vol. 76, pp. 1-16, 2015.

[40] H. Hayashi, "Standardization of wireless coexistence in industrial automation", in 2015 54th Annual Conference of the Society of Instrument and Control Engineers of Japan (SICE), pp. 968-973, 2015.

[41] T. M. Chiwewe, C. F. Mbuya, and G. P. Hancke, "Using Cognitive Radio for Interference-Resistant Industrial Wireless Sensor Networks: An Overview," IEEE Trans. on Industrial Informatics, vol. 11, no. 6, pp. 1466-1481, 2015.

[42] P De Valck, I Moerman, D Croce, F Giuliano, I Tinnirello, D Garlisi, E. De Poorter, B. Jooris, "Exploiting programmable architectures for WiFi/ZigBee inter-technology cooperation”, EURASIP Journal on Wireless Communications and Networking 2014.

[43] Kameswari Chebrolu and Ashutosh Dhekne. "Esense: communication through energy sensing". In Proceedings of the 15th annual international conference on Mobile computing and networking (MobiCom '09). ACM, New York, NY, USA, 85-96, 2009.

[44] A. Fischer, J. Botero, M. Till Beck, H. de Meer, X. Hesselbach, "Virtual Network Embedding: A Survey”, IEEE Communications Surveys \& Tutorials, vol. 15, no. 4, 2013.

[45] T. Lin, H. Bannazadeh, A. Leon-Garcia, "Introducing wireless access programmability using software-defined infrastructure", IFIP/IEEE International Symposium on Integrated Network Management (IM), pp. 585-591, 2015.

[46] Guo, K., Sanadhya, S., Woo, T., "Vifi: virtualizing wlan using commodity hardware", ACM SIGMOBILE Mobile Computing and Communications review, vol. 18 , no. 3 , pp. 41-48, 2015.

[47] A. Pelov, L. Toutain, Y. Delibie, "Constrained Signaling over LP-WAN", Internet Engineering Task Force draft, https://tools.ietf.org/html/draft-pelovcore-cosol-01, February 17, 2016.

[48] F. Van den Abeele, J. Hoebeke, I. Moerman and P. Demeester, "Fine-grained management of CoAP interactions with constrained IoT devices", IEEE/IFIP Network Operations and Management Symposium, Proceedings. pp.1-5, 2014.

[49] Bonomi, Flavio, et al. "Fog computing and its role in the internet of things." Proceedings of the first edition of the MCC workshop on Mobile cloud computing. ACM, Pages 13-16, Helsinki, Finland, August 17, 2012.

[50] Hu, Y. C., Patel, M., Sabella, D., Sprecher, N., \& Young, V., "Mobile edge computing_A key technology towards 5G", ETSI White Paper (11), 2015. 
[51] Baldo, N.; Tamma, B.R.; Manoj, B.; Rao, R.; Zorzi, M. ,"A neural network based cognitive controller for dynamic channel selection". Communications, 2009. ICC'09. IEEE International Conference on. IEEE,2123 2009, pp. 1-5..

[52] Zorzi, M.; Zanella, A.; Testolin, A.; De Filippo De Grazia, M.; Zorzi, M. "Cognition-Based Networks: A 2130 New Perspective on Network Optimization Using Learning and Distributed Intelligence". Access, IEEE 2131 2015, 3, 15121530.

[53] M. Kulin, C. Fortuna, E. De Poorter, D. Deschrijver and I. Moerman, "Datadriven design of intelligent wireless networks: an overview and tutorial", Sensors Journal, 16(6), 790, 2016.

[54] Randy Compton, Michael T. Mehari, Charles J. Colbourn, Eli De Poorter, Violet R. Syrotiuk, "Screening Interacting Factors in a Wireless Network Testbed Using Locating Arrays", The International Workshop on Computer and Networking Experimental Research, IEEE Conference on Computer Communications Workshops (INFOCOM WKSHPS), 2016.

[55] W. Munawar , M. H. Alizai , O. Landsiedel , K. Wehrle, "Dynamic TinyOS: Modular and Transparent Incremental Code-Updates for Sensor Networks", IEEE ICC 2010, May 23-27.

[56] P. J. Marrón, M. Gauger, A. Lachenmann, D. Minder, O. Saukh, and K. Rothermel, "FlexCup: A Flexible and Efficient Code Update Mechanism for Sensor Networks", EWSN 2006, February 13-15.

[57] Ruckebusch, P., De Poorter, E., Fortuna, C., \& Moerman, I., "GITAR: Generic extension for Internet-of-Things ARchitectures enabling dynamic updates of network and application modules". Ad Hoc Networks, Volume 36 Issue P1, January 2016.

[58] I. Ishaq, J. Hoebeke, I. Moerman and P. Demeester, “Observing CoAP groups efficiently”, Ad Hoc Networks, vol.37, part 2, pp. 368-388, Feb. 2016.

[59] I. Ishaq, J. Hoebeke, F. Van den Abeele, J. Rossey, I. Moerman and P. Demeester, "Flexible Unicast-Based Group Communication for CoAP-Enabled Devices", Sensors, 14(60), pp. 9833-9877, 2014. 